\title{
REVESTIMENTOS DE ALTA CAMADA OBTIDOS COM A MODIFICAÇÃO DE RESINAS EPÓXI NOVOLACA PELA REAÇÃO COM ORGANO-SILOXANO E CATÁLISE ÁCIDA
}

\author{
Roberta Jachura Rocha*, José Atílio Fritz Fidel Rocco, Maria Auxiliadora Silva de Oliveira e Koshun Iha \\ Departamento de Química, Instituto Tecnológico de Aeronáutica, 12228-900 São José dos Campos - SP, Brasil
}

Recebido em 13/06/2016; aceito em 12/09/2016; publicado na web em 12/10/2016

\begin{abstract}
HIGH LAYER COATING OBTAINED IN THE REACTION OF MODIFIED NOVOLAC EPOXYS RESINS WITH ORGANOSILOXANES THROUGH ACID CATALYSIS. Epoxy resins are an important class of thermostable polymers, widely used in structural applications or as adhesives. However, the low fracture resistance is their main weakness in many applications. Due to this fact, this study aims to modify a polymer matrix based on a novolac epoxy resin with an organo siloxane (AMS). The final properties of cured epoxys resins are affected by the curing process and the coatings modified in this study with AMS and cured with acid catalyst presented greater resistance to thermal decomposition compared to that cured with Aradur, demonstrating a higher content of crosslinking in the siloxane polyether and the novolak epoxy resin chains. This epoxy matrix modified was used to prepare coatings whose films applied to 1020 steel surfaces presented a resistance of $10^{10} \mathrm{Ohm}-\mathrm{cm}^{-2}$. This resistance value is one order of magnitude greater than that of epoxy resins films modified by siloxanes in published studies, which demonstrates a potential economy in terms of maintenance costs of metal structures and machines used in power plants (UTE). To obtain the developed inks in pilot plant scale, it was used an aromatic polyamine as curing agent, characterizing the product obtained as an epoxy bi-component paint for commercial use.
\end{abstract}

Keywords: Corrosion, novolac epoxy resin, interpenetrating polymer network-IPN, polyether siloxane, coatings.

\section{INTRODUÇÃO}

As resinas epóxi são uma importante classe de polímeros termoestáveis, amplamente utilizadas em aplicações estruturais ou como adesivos, devido a suas características de alta tensão e módulo, fácil processamento, boa resistência química e térmica e alta aderência a muitos substratos. Os sistemas epóxi apresentam pequena contração após a cura (em média, 2\%), o que indica a necessidade de um baixo grau de rearranjo molecular para formar o sistema curado. Quanto à estabilidade química, o epóxi é um dos termorrígidos mais inertes, além de possuir boa estabilidade dimensional. Entretanto, revestimentos à base de resinas epóxi apresentam hidrofobicidade, baixas resistência ao impacto e à exposição a ambientes abertos, estabilidade térmica relativamente baixa e, em muitas aplicações, sua principal desvantagem é sua baixa resistência à fratura. Por essas razões, as resinas epóxi são frequentemente modificadas buscando aprimorar suas características.

Conforme estudo de Gu et al., ${ }^{1}$ têm sido introduzidas nanopartículas inorgânicas $\left(\mathrm{SiO}_{2}, \mathrm{ZrO}_{2}, \mathrm{ZnO}\right)$ em matrizes epóxi que atuam preenchendo os defeitos, como microfissuras e vazios, do revestimento epóxi, com o intuito de aprimorar suas propriedades anticorrosivas modificando seu efeito como barreira física. A otimização de propriedades, principalmente mecânicas, das resinas epóxi e dos polímeros termofixos também pode ser alcançada pela modificação da estrutura do polímero, aumentando-se a cadeia em pontos de entrecruzamento. ${ }^{2}$ De forma geral, a introdução de segmentos poliméricos flexíveis à cadeia de resinas epóxi melhora sua resistência ao impacto, enquanto mantém as demais propriedades praticamente inalteradas. A incorporação de polisiloxanos elásticos à cadeia de resinas epóxi também pode resultar em aumento nos valores de propriedades físico-químicas como hidrofobicidade, resistência à erosão, resistência à exposição a ambientes abertos (UV), resistência a solventes e produtos químicos em geral, resistência à corrosão e estabilidade termo-oxidativa, entre outras. ${ }^{1}$

\footnotetext{
*e-mail: robertajr@gmail.com
}

Dentre as formas de incorporação de siloxanos/silicone a polímeros, o mecanismo que permite a formação de estruturas poliméricas interpenetrantes (Interpenetrating Polymer Networks - IPNs) proporciona a estabilidade de propriedades específicas que possibilitam ampliar o número de aplicações dos produtos assim modificados. ${ }^{2-6}$

Numa estrutura polimérica interpenetrante ideal, duas ou mais estruturas poliméricas, quimicamente distintas e com elevado grau de ligações cruzadas dentro de suas cadeias, são mantidas unidas exclusivamente por seu entrelaçamento. No entanto, podem ocorrer situações em que somente um dos componentes apresente ligações cruzadas em sua estrutura polimérica ou, ainda, situações em que as ligações internas das cadeias poliméricas dos componentes não estão entrelaçadas, o que pode ocasionar separação de fases. ${ }^{7}$ Neste estudo, buscou-se sintetizar uma estrutura polimérica baseada no método convencional no qual as ligações cruzadas ocorrem dentro das respectivas cadeias poliméricas e entre cada cadeia polimérica que constitui a estrutura interpenetrante. Os pré-polímeros selecionados são uma resina epóxi novolaca e um poliéter siloxano.

As resinas epóxi novocala, cuja estrutura química está apresentada na Figura 1, são sintetizadas pela reação de uma resina fenólica novolaca com epicloridrina. Os vários grupos epóxi presentes nas resinas epóxi novolaca contribuem para suas altas densidades de reticulação, o que resulta em excelentes propriedades de resistência química, térmica e a solventes ${ }^{8,9}$

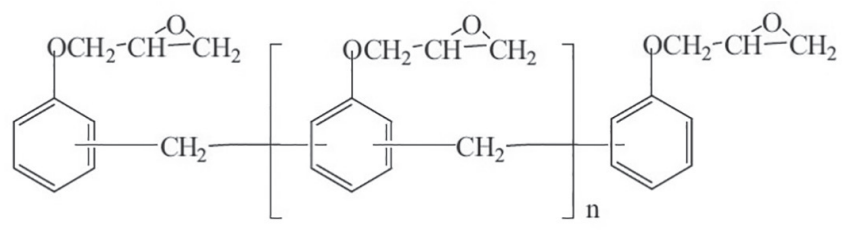

Figura 1. Estrutura química da resina epóxi novolaca ${ }^{10}$

O poliéter siloxano apresenta grupos siloxano terminais e sua introdução proporciona flexibilidade, elongação, aumenta a resistência 
à abrasão e ablação sob calor e melhora a integridade de materiais utilizados para preparar filmes para recobrimento de superfícies. Sua estrutura química está apresentada na Figura 2.

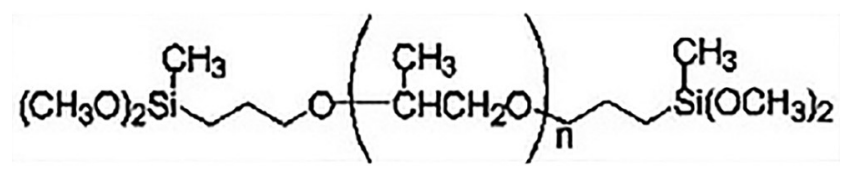

Figura 2. Estrutura química do polieter siloxano (Silyl)

Sabe-se, também, que as propriedades finais das resinas epóxi são influenciadas pelo processo de cura e dependem da combinação específica do tipo de resina epóxi e do agente de cura utilizados..$^{10-13}$ Neste estudo, foi realizada uma catálise ácida para posterior cura da mistura pré-hidrolizada obtida, fato que torna único o método proposto quando comparado aos métodos convencionais de produção de resinas modificadas com siloxano, nos quais ocorre catálise alcalina com o emprego de aminas, amino siloxanos e compostos organometálicos.

\section{PARTE EXPERIMENTAL}

O método desenvolvido para a modificação da resina epóxi novolaca por incorporação de siloxanos, com base no método convencional de formação da estrutura IPN, emprega um alquil-amino siloxano como agente de compatibilização para a mistura de siloxano e resina epóxi.

$\mathrm{Na}$ formação da IPN pelo método convencional, ocorrem reações de hidrólise dos grupos alcoxi $\left(\mathrm{OC}_{\mathrm{n}} \mathrm{H}_{(2 \mathrm{n}+1)}\right)$ do organo-siloxano, formando grupos silanol ( $\mathrm{Si}-\mathrm{OH})$ que podem condensar e formar estruturas - $[\mathrm{Si}-\mathrm{O}-\mathrm{Si}] .{ }^{2}$ Essas estruturas podem, paralelamente, reagir com os grupos $\mathrm{C}-\mathrm{OH}$ formados na abertura do anel epóxi em sua reação com o alquil-amino siloxano, levando à formação de estruturas - $[\mathrm{Si}-\mathrm{O}-\mathrm{Si}]_{\mathrm{n}}-\mathrm{Si}-\mathrm{O}-\mathrm{C}-{ }^{4,14-20}$ Porém, para determinadas aplicações, o filme epóxi-siloxano obtido por esse método convencional se apresenta pouco flexível, quebradiço e com baixa resistência à abrasão e ao impacto. Para melhorar essas propriedades, a variação feita neste estudo tem o propósito de utilizar uma quantidade de alquil-amino siloxano suficiente apenas para realizar a pré-abertura dos anéis epóxi da resina novolaca e agir como catalisador para a pré-hidrólise do poliéter siloxano, conforme reação global apresentada na Figura 3. Conforme citado, realizou-se uma catálise ácida para posterior cura da mistura pré-hidrolizada obtida.

Em um reator aberto, exposto ao ar atmosférico e sem aquecimento, foram postas a reagir partes iguais da resina epóxi novolaca Araldite EPN 1179 e do poliéter siloxano (Silyl) do tipo (XO) $)_{2} \mathrm{SiY}$ produzido pela Kaneka of Japan, em que XO é um grupo metoxi $\left(\mathrm{OCH}_{3}\right)$ hidrolizável e Y é um grupo organo-funcional $\left(\mathrm{CH}_{3}\right)$. Adicionou-se cerca de $1 \% \mathrm{~m} / \mathrm{m}$ de água e catalisador ácido para promover a pré-hidrólise da mistura, mantida sob agitação mecânica por cerca de 30 minutos na temperatura ambiente $\left(\sim 25^{\circ} \mathrm{C}\right)$. Também foi adicionado à mistura cerca de $20 \%(\mathrm{~m} / \mathrm{m})$ de dióxido de titânio $\left(\mathrm{TiO}_{2}\right)$, mantendo a agitação por quatro horas adicionais. Após esse processo, a tinta obtida foi denominada de "Tinta base seca". Numa segunda fase, foi incorporado o equivalente a $20 \%(\mathrm{~m} / \mathrm{m})$ de solvente orgânico (xilenol e metanol) (50\% v/v), obtendo-se uma tinta denominada de "Tinta base úmida".

Para o princípio envolvido na variação do método convencional citado, em um reator aberto, exposto ao ar atmosférico e sem aquecimento, foram postas a reagir partes iguais da resina epóxi novolaca EPN 1179 e do Silyl, e uma quantidade de alquil-amino siloxano (AMS), produzido pela Sigma-Aldrich, suficiente para promover a pré-abertura dos anéis epóxi e a pré-hidrólise da resina epóxi e do siloxano. A mistura reagente foi mantida sob agitação mecânica por cerca de 30 minutos. Tomou-se uma porção da mistura resultante e adicionaram-se cerca de $20 \%(\mathrm{~m} / \mathrm{m})$ do $\mathrm{TiO}_{2}$, mantendo a mistura obtida sob agitação por mais quatro horas. A tinta obtida foi denominada de "Tinta modificada com AMS-Base seca-1". De forma semelhante à preparação da "Tinta base úmida" anteriormente descrita, uma quarta tinta, denominada "Tinta modificada com AMS-Base úmida-1", foi preparada utilizando uma porção da mistura da resina epóxi novolaca EPN 1179 e do Silyl submetida à pré-hidrólise catalisada pelo AMS.

Outras duas tintas modificadas com AMS, denominadas "Tinta modificada com AMS-Base seca-2" e "Tinta modificada com AMSBase úmida-2", foram preparadas utilizando uma porção da mistura da resina epóxi novolaca EPN 1179 e do Silyl submetida à pré-hidrólise catalisada pelo AMS com a incorporação de $10 \%(\mathrm{~m} / \mathrm{m})$ de $\mathrm{TiO}_{2}$ e $50 \%(\mathrm{~m} / \mathrm{m})$ de $\mathrm{SiO}_{2}$, além de $20 \% \mathrm{~m} / \mathrm{m}$ do solvente no caso da "Tinta modificada com AMS-Base úmida-2”.
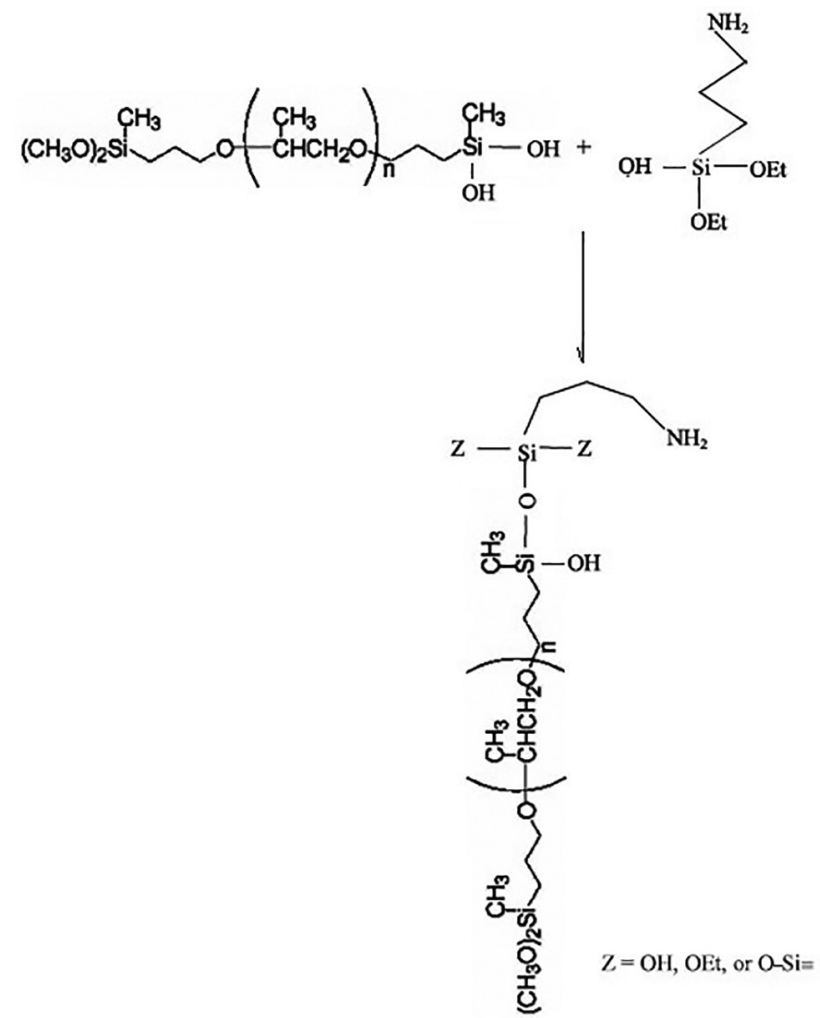

Figura 3. Reação de condensação do AMS hidrolisado com um grupo $\mathrm{Si-OH}$ do poliéter siloxano hidrolisado. Adaptado de Oteo et al. ${ }^{21}$

As tintas obtidas foram utilizadas como revestimento de superfícies de corpos de prova metálicos feitos a partir de uma chapa do aço 1020, material bastante empregado em tubulações e tanques. Estudou-se a decomposição térmica de algumas das tintas desenvolvidas pelo método termogravimétrico (TG) com o uso do Analisador Termogravimétrico Shimadzu TGA-51, sob atmosfera dinâmica de nitrogênio $\left(50 \mathrm{~mL} \mathrm{~min}{ }^{-1}\right)$ e razão de aquecimento de $20^{\circ} \mathrm{C} \mathrm{min}^{-1}$, iniciando-se o aquecimento em temperatura ambiente $\left(\sim 25^{\circ} \mathrm{C}\right)$ até as temperaturas de $600{ }^{\circ} \mathrm{C}$ e $1000{ }^{\circ} \mathrm{C}$. Na técnica de calorimetria exploratória diferencial (DSC), fez-se uso do Analisador Shimadzu DSC 60 empregando atmosfera dinâmica de nitrogênio $\left(50 \mathrm{~mL} \mathrm{~min}{ }^{-1}\right)$, partindo da temperatura ambiente, utilizando células de alumínio e de platina nos ensaios em que a temperatura foi elevada até $600{ }^{\circ} \mathrm{C} \mathrm{e}$ $1000{ }^{\circ} \mathrm{C}$, respectivamente, a uma velocidade de $20^{\circ} \mathrm{C} \mathrm{min}{ }^{-1}$.

Além disso, os produtos obtidos foram caracterizados por absorção de radiação no infravermelho (FTIR), com o emprego do 
espectrofotômetro Varian 660-IR equipado com sistema de reflexão ATR (Attenuated Total Reflection). Os espectros foram obtidos na faixa de $4000-4520 \mathrm{~cm}^{-1}$, com resolução de $4 \mathrm{~cm}^{-1}$ e um total de 16 varreduras acumuladas nas aquisições de cada espectro.

Foram realizadas também análises do potencial de corrosão (Ecorr) das superfícies recobertas com as tintas desenvolvidas neste estudo, que demonstra a tendência de as superfícies recobertas com as tintas sofrerem corrosão, e da densidade de corrente de corrosão (Jcorr), que mostra a eficiência de proteção contra corrosão ( $\mathrm{P} \%)$ oferecida pelas tintas, determinada pela equação 1 :

$$
P \%=100 \times\left(1-\frac{J_{\text {corr }}}{J_{\text {corr }}}\right)
$$

em que Jcorr é a densidade de corrente de corrosão das superfícies recobertas com as respectivas tintas, e J corr $_{o}$ é a densidade de corrente de corrosão do substrato metálico sem o filme.

Para isso, foram recobertas superfícies de corpos de prova metálicos feitos de aço carbono 1020, previamente jateadas com areia na condição de metal quase ao branco, para uma primeira série de experimentos com quatro demãos da "Tinta base úmida" curada com um catalisador ácido, e com quatro demãos da "Tinta modificada com AMS-Base úmida-1" para uma segunda série de experimentos. Em seguida, as superfícies recobertas foram submetidas a ensaios eletroquímicos de corrosão, com polarização potenciodinâmica e espectroscopia de impedância eletroquímica (EIS) em um meio aquoso contendo cloreto, solução aquosa $0,5 \mathrm{~mol} \mathrm{~L}^{-1}$ de $\mathrm{NaCl}$ e pH igual a 5,8, exposta à atmosfera ambiente, sem agitação, na temperatura ambiente. Estes ensaios foram realizados empregando-se o potenciostato/galvanostato PGSTAT 30 da AUTOLAB conectado a um computador tipo PC através da interface USB - IF030 e controlado pelos programas GPES.EXE (ensaios potenciodinâmicos) e FRA.EXE (EIS). Foi utilizada uma célula eletroquímica com três eletrodos: (1) eletrodo de trabalho: superfícies pintadas e superfícies denominadas de branco com área exposta de $0,502 \mathrm{~cm}^{-2}$; (2) eletrodo auxiliar: fio de platina em espiral; (3) eletrodo de referência: eletrodo de $\mathrm{Ag} / \mathrm{AgCl}, \mathrm{Cl}^{-}$saturado. Todos os ensaios foram realizados em triplicata. ${ }^{22,23}$

\section{RESULTADOS E DISCUSSÃO}

\section{Análises dos espectros de Radiação de Absorção no Infravermelho (FTIR)}

A região mais importante nos espectros FTIR de polisiloxanos compreende a faixa de número de onda entre 1000-1225 $\mathrm{cm}^{-1}$, correspondente a vibrações de estiramento das ligações $\mathrm{Si}-\mathrm{O}-\mathrm{C}$ e $\mathrm{Si}-\mathrm{O}-\mathrm{Si}$ nas estruturas formadas pela condensação dos grupos silanóis (Si-OH) gerados na hidrólise de alcoxisilanos. Nesta região, os espectros costumam apresentar máximos em 1012 e $1045 \mathrm{~cm}^{-1}$, atribuídos a vibrações de ligações $\mathrm{Si}-\mathrm{O}-\mathrm{Si}$ em cadeias lineares, e máximo em $1094 \mathrm{~cm}^{-1}$, com um ombro ao redor de $1086 \mathrm{~cm}^{-1}$, atribuído a vibrações de ligações $\mathrm{Si}-\mathrm{O}-\mathrm{Si}$ (vas $\mathrm{Si}-\mathrm{O}-\mathrm{Si}$ ) em cadeias com um número relativamente baixo de ligações cruzadas. Os espectros FTIR de polisiloxanos também costumam apresentar máximos em 1144, 1174 e $1198 \mathrm{~cm}^{-1}$, atribuídos a estruturas contendo anéis cíclicos com 4, 5 e 6 membros, respectivamente. ${ }^{24-26}$ Outra banda de absorção importante está relacionada a vibrações da ligação $\mathrm{Si}-\mathrm{OH}$ em $\sim 950 \mathrm{~cm}^{-1} .^{27-29}$

No caso das resinas epóxi novolaca, entre os vários picos de absorção que podem ser observados em seus espectros FTIR, o de maior importância é o pico em $\sim 912 \mathrm{~cm}^{-1}$ referente ao anel epóxi. Outros picos importantes situam-se em $\sim 3000 \mathrm{~cm}^{-1}$, atribuído ao estiramento $\mathrm{CH}$ no anel epóxi, pico em $1509 \mathrm{~cm}^{-1}$, atribuído ao estiramento das ligações $\mathrm{C}=\mathrm{C}$ dos anéis benzênicos, pico em $\sim 1238$ $\mathrm{cm}^{-1}$, atribuído principalmente ao estiramento da ligação éter anel aromático-oxigênio, e o pico em $\sim 1030 \mathrm{~cm}^{-1}$, atribuído à vibração do grupo $-\mathrm{OH}_{2}-.^{30-34}$

Para a mistura da resina epóxi novolaca EPN1179 com o poliéter siloxano submetida à pré-hidrólise com catálise ácida, fez-se uma análise detalhada dos espectros FTIR (Figura 5) nas regiões correspondentes às absorções do estiramento do anel epóxi, em $3001 \mathrm{~cm}^{-1} \mathrm{e} 912 \mathrm{~cm}^{-1}$; e correspondente às vibrações do estiramento de ligações $\mathrm{Si}-\mathrm{O}-\mathrm{Si}$ formadas na condensação dos grupos silanóis (Si-OH), em 1000-1225 cm-1, que também podem estar relacionadas às vibrações de ligações $\mathrm{Si}-\mathrm{O}-\mathrm{C}$ pré-existentes e/ou formadas na

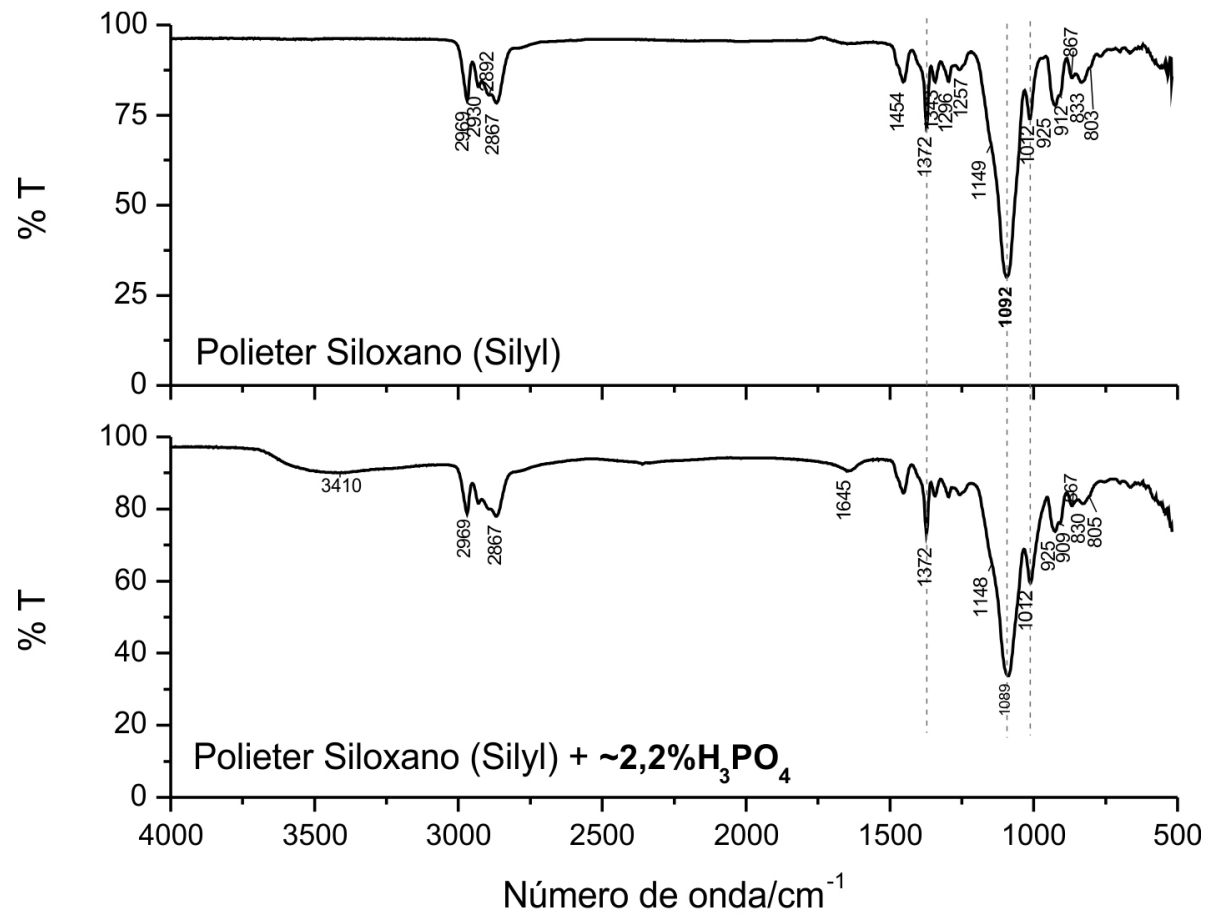

Figura 4. FTIR para o poliéter siloxano antes e após a cura catalisada por ácido 
reação de condensação entre silanóis com os grupos $\mathrm{C}-\mathrm{OH}$ oriundos da abertura de anéis epóxi.

A partir dessa análise, conclui-se que ocorre a abertura parcial de anéis epóxi tanto na mistura pré-hidrolisada da resina epóxi novolaca EPN1179 com o poliéter siloxano, como nas tintas não curadas preparadas a partir dessa mistura, uma vez que se nota o desaparecimento do pico de absorção atribuído ao estiramento $\mathrm{CH}$ no anel epóxi em $3001 \mathrm{~cm}^{-1}$.

Há, também, indícios de crescimento da cadeia polimérica da resina epóxi novolaca pela reação de homopolimerização, na qual um grupo $\mathrm{C}-\mathrm{OH}$ do glicol, produzido na reação de adição de uma molécula de água a um anel epóxi protonado, liga-se a outro anel epóxi protonado formando um éter de cadeia maior. Esse fato pode ser indicado pelo pico de absorção na região de $1030 \mathrm{~cm}^{-1}$ no espectro ATR/FTIR da novolaca pura, atribuído ao estiramento da ligação éter do grupo - $-\mathrm{CH}_{2}-$, que aparece deslocado para regiões de números de ondas um pouco mais elevados nos espectros FTIR da mistura pré-hidrolisada e das tintas não curadas preparadas a partir dessa mesma mistura, com ombros/picos entre 1035 e $1041 \mathrm{~cm}^{-1}$. Para justificar o aparecimento destes ombros/picos, pode-se considerar a contribuição adicional da absorção de grupos $-\mathrm{CH}_{2}-\mathrm{OH}$ formados na reação de homopolimerização da resina. ${ }^{32}$

Por outro lado, a ausência do pico em $\sim 950 \mathrm{~cm}^{-1}$, atribuído a vibrações de ligações $\mathrm{Si}-\mathrm{OH}$, nos espectros das tintas não curadas contendo o poliéter siloxano, e o aparecimento de um ombro relativamente intenso na região de $1054 \mathrm{~cm}^{-1}$, que pode estar relacionado a vibrações de ligações $\mathrm{Si}-\mathrm{O}-\mathrm{Si}$ em cadeias lineares de polisiloxanos, e/ou à formação de ligações de condensação de grupos $-\mathrm{CH}_{2}-\mathrm{OH}$ produzidos na abertura do anel epóxi com um grupo silanol ( $\mathrm{Si}-\mathrm{OH})$ gerado na pré-hidrólise do poliéter siloxano, sugerem a formação de uma estrutura muito maior com ligação de cruzamento entre os dois polímeros.

Os indícios de ligações cruzadas entre os grupos $-\mathrm{Si}-\mathrm{OH}$ e $-\mathrm{CH}_{2}-$ $\mathrm{OH}$ nas estruturas poliméricas formadas na pré-hidrólise são ainda mais fortes nos espectros FTIR das tintas não curadas. Nestes espectros, observa-se um deslocamento do pico em $1092 \mathrm{~cm}^{-1}$, atribuído ao modo vibracional vas $\mathrm{Si}-\mathrm{O}-\mathrm{C}$ de ligações dos grupos metoxi com átomos de $\mathrm{Si}^{35-37}$ nos espectros FTIR do poliéter siloxano puro e da mistura pré-hidrolisada, para regiões de número de onda sistematicamente maiores $\left(\sim 1097 \mathrm{~cm}^{-1}\right)$.

Nos espectros FTIR de tintas não curadas, observa-se uma diminuição no valor da relação entre a absorbância do pico em 1097 $\mathrm{cm}^{-1} \mathrm{e}$ da absorbância do pico em $1509 \mathrm{~cm}^{-1}$, atribuído ao estiramento das ligações $\mathrm{C}=\mathrm{C}$ dos anéis benzênicos presentes na resina epóxi novolaca e tomado como referência pelo fato de os anéis aromáticos não serem significativamente afetados pela reação de cura, em relação à razão semelhante para o pico em $\sim 1092 \mathrm{~cm}^{-1}$ no espectro FTIR da mistura resina epóxi novolaca EPN1179 com poliéter siloxano pré-hidrolisada, em que o deslocamento mencionado para é ainda maior nos espectros FTIR das tintas base seca e base úmida curadas ( 1102 $\left.\mathrm{cm}^{-1}\right)$. Portanto, o valor da relação entre a absorbância do pico em $\sim 1102 \mathrm{~cm}^{-1}$ e a absorbância do pico tomado como referência (1509 $\mathrm{cm}^{-1}$ ) nos espectro FTIR de ambas as tintas base seca e base úmida curadas é cerca de 50\% menor do que o valor da relação semelhante para o pico em $\sim 1092 \mathrm{~cm}^{-1}$ nos espectros FTIR da mistura resina epóxi novolaca EPN1179/ poliéter siloxano pré-hidrolisada, e cerca de $30 \%$ menor do que o valor da relação semelhante para os mesmos picos nos espectros das tintas não curadas.

O menor valor desta relação sugere uma diminuição na quantidade de ligações $\mathrm{Si}-\mathrm{O}-\mathrm{C}$ das tintas base seca e base úmida curadas, o que pode ser decorrente do processo de hidrólise dos grupos metoxi do poliéter siloxano e subsequente condensação entre os grupos silanóis $(\mathrm{Si}-\mathrm{OH})$ formados, e entre grupos silanóis e grupos $-\mathrm{CH}_{2}-$ $\mathrm{OH}$ produzidos na abertura do anel epóxi, o que também justifica o deslocamento que ocorre na banda atribuída a vas Si-O-Si e a vas $\mathrm{Si}-\mathrm{O}-\mathrm{C}$, em números de ondas mais elevados, nos espectros das tintas base seca e base úmida curadas e não curadas. Neste ponto, ressalta-se que a banda correspondente a vibrações $\mathrm{Si}-\mathrm{OH}\left(\sim 950 \mathrm{~cm}^{-1}\right)$ não

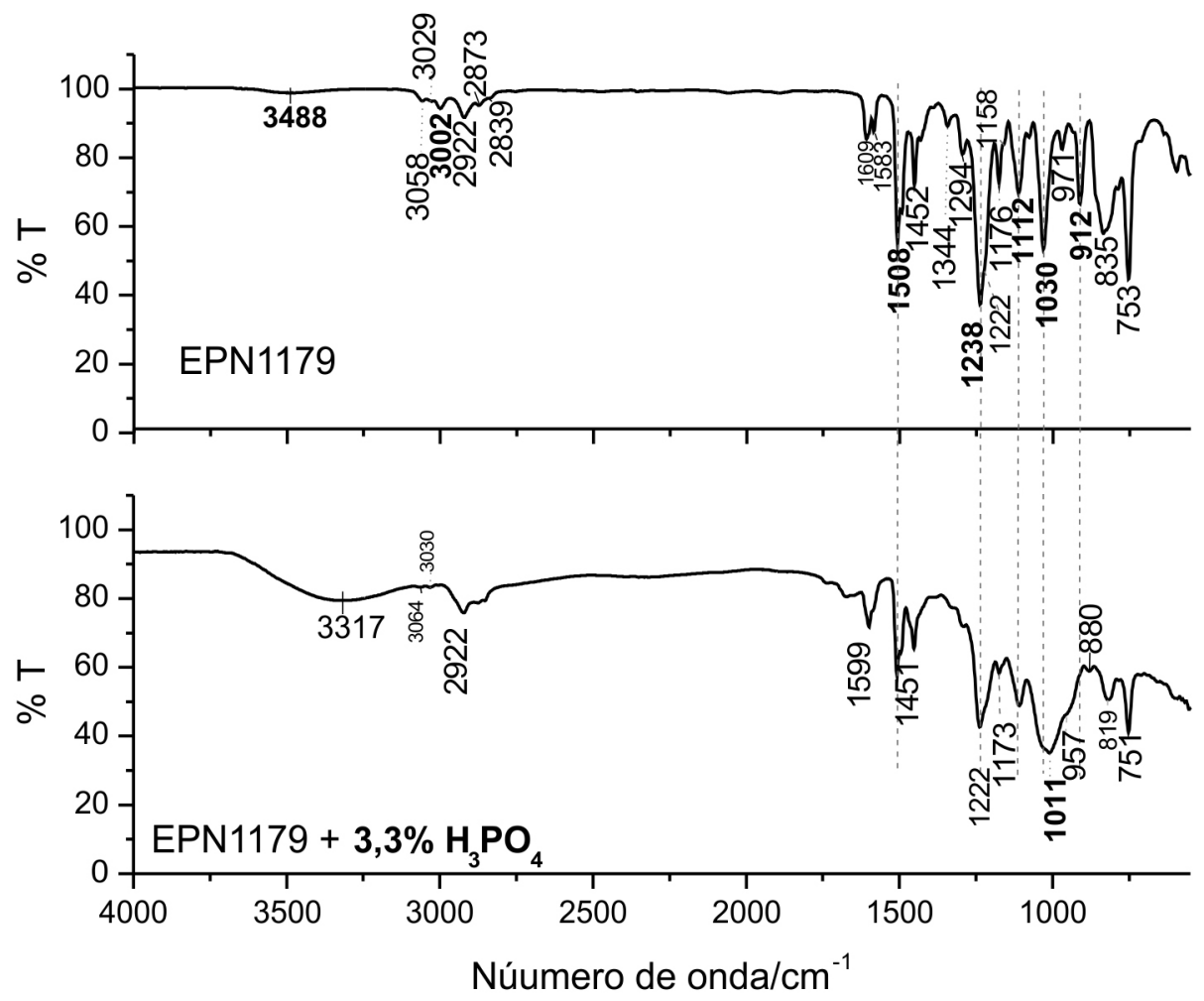

Figura 5. FTIR para a resina epóxi EPN1179 antes e após a cura catalisada por ácido 
é observada nos espectros FTIR das Tinta base seca e base úmida curadas e não curadas ou das misturas pré-hidrolisadas.

Adicionalmente, nota-se nos espectros das Tintas modificadas com AMS curadas que os valores para a relação entre as absorbâncias do pico em $1014 \mathrm{~cm}^{-1}$ (atribuído a vibrações de ligações Si-O-Si em cadeias lineares, vas $-\mathrm{HC}-\mathrm{O}-\mathrm{CH} 2-,{ }^{31,34}$ hidroxilas alifáticas ${ }^{32}$ ) e do pico em $\sim 1035 \mathrm{~cm}^{-1}$ (atribuído à vibração do grupo -O-CH2-), e a absorbância do pico tomado como referência, são cerca de $50 \%$ maiores comparados aos valores da relação semelhante para esses picos nos espectros das tintas base seca e úmida curadas. Esse fato indica a influência da carga adicional $\left(\mathrm{SiO}_{2}\right)$ no processo de formação da estrutura polimérica interpenetrante e permite sugerir que, nas tintas modificadas com AMS, o número de ligações cruzadas na cadeia do polisiloxano (pico em $\sim 1085 \mathrm{~cm}^{-1}$ ) e de ligações éter e hidroxilas alifáticas na cadeia da resina epóxi (pico em $1014 \mathrm{~cm}^{-1}$ ) aumentam. Desta forma, sugere-se a formação de duas cadeias poliméricas entrelaçadas com um número relativamente maior de ligações cruzadas nas cadeias individuais. Ao mesmo tempo, o aumento do valor da relação entre a absorbância do pico em $\sim 1055 \mathrm{~cm}^{-1}$ e do pico tomado como referência, em $1509 \mathrm{~cm}^{-1}$, sugere uma maior estrutura polimérica das tintas modificadas com AMS curadas, devido ao maior teor de ligações de condensação de grupos $-\mathrm{CH}_{2}-\mathrm{OH}$ gerados na abertura do anel epóxi, com grupos silanol gerados na pré-hidrólise do poliéter siloxano.

\section{Análises de Termogravimetria (TG) e Calorimetria Exploratória Diferencial (DSC)}

Os resultados das análises térmicas (TG e DSC) realizadas para amostras da "Tinta base seca" curada com poliamina aromática (Figura 6) evidenciam que a decomposição térmica ocorre em, pelo menos, quatro etapas distintas. Na primeira etapa, partindo da temperatura ambiente até $125^{\circ} \mathrm{C}$, ocorre um processo endotérmico que pode ser atribuído à perda da água adsorvida na estrutura polimérica interpenetrante. Em uma segunda etapa, entre as temperaturas de 125 a $295{ }^{\circ} \mathrm{C}$, ocorre perda de massa de cerca de $8 \%(\mathrm{~m} / \mathrm{m})$, que pode estar relacionada à banda larga de diminuição do fluxo de calor que aparece na curva DSC, indicando processo endotérmico e possível decomposição térmica da amostra, associada a uma reorganização estrutural das cadeias poliméricas interpenetrantes.

Observa-se uma perda de massa adicional de cerca de $52 \%(\mathrm{~m} / \mathrm{m})$ na faixa de temperatura entre 295 a $480{ }^{\circ} \mathrm{C}$, na qual a curva DSC se caracteriza pelo aparecimento de dois picos exotérmicos de fluxo de calor $\left(406\right.$ e $449^{\circ} \mathrm{C}$ ) e, adicionalmente, no mínimo dois ombros com fluxo positivo de calor em 331 e $362{ }^{\circ} \mathrm{C}$, o que caracteriza eventos exotérmicos. O processo exotérmico associado ao pico em $406{ }^{\circ} \mathrm{C}$ foi identificado como a decomposição térmica do poliéter siloxano utilizado em excesso estequiométrico. A presença de excesso desse componente é benéfica, pois possibilita uma posterior absorção de quantidades residuais de umidade, mantendo a integridade estrutural do filme. Por outro lado, o processo que ocorre na faixa de 420 a $480{ }^{\circ} \mathrm{C}$ pode estar associado à desidratação, pela eliminação de água de grupos $-\mathrm{CH}_{2}-\mathrm{CH}(\mathrm{OH})-$ da resina epóxi novolaca e formação subsequente de duplas ligações. ${ }^{38}$

Para temperaturas entre 480 a $750^{\circ} \mathrm{C}$, a perda de massa é de cerca de $15 \%(\mathrm{~m} / \mathrm{m})$. Nesta faixa de temperatura, a curva DTG (Figura 7$)$, derivada primeira da curva TG, apresenta um pico em $675^{\circ} \mathrm{C}$ que pode estar relacionado a dois ombros com fluxo de calor positivo, processos exotérmicos, observados em temperaturas acima de $480^{\circ} \mathrm{C}$. Em estruturas híbridas de siloxanos e resinas epóxi novolaca curadas com amina, notam-se picos de decomposição térmica em temperaturas elevadas $\left(520\right.$ a $\left.690^{\circ} \mathrm{C}\right)$ que têm sido atribuídos à decomposição térmica de cadeias poliméricas aprisionadas na estrutura inorgânica

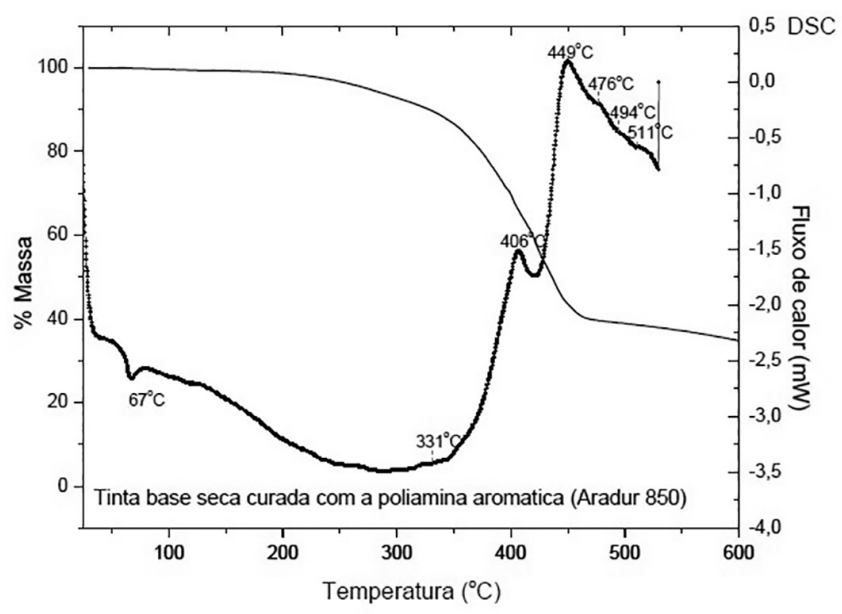

Figura 6. Curvas TG e DSC para a Tinta base seca curada com a poliamina aromática (Aradur 850) em atmosfera de $\mathrm{N}_{2}$

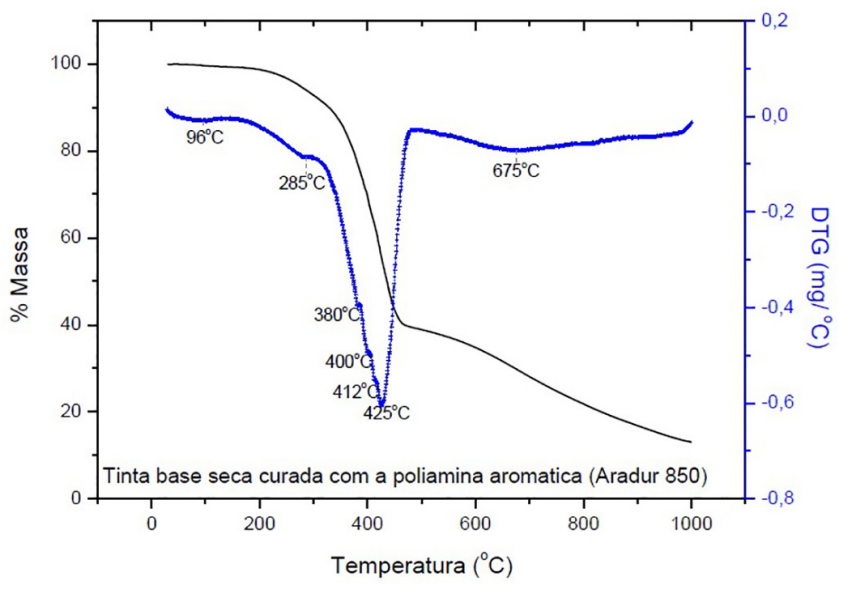

Figura 7. Curvas TG e DTG para a Tinta base seca curada com a poliamina aromática (Aradur 850) em atmosfera de $\mathrm{N}_{2}$

formada. ${ }^{38}$ Esta faixa de temperatura inclui o pico observado em 675 ${ }^{\circ} \mathrm{C}$ na curva DTG da "Tinta base seca" curada com o Aradur 850, podendo, desta forma, ser associado a evento semelhante. A massa residual na temperatura de $750{ }^{\circ} \mathrm{C}$ é de cerca de $25 \%$ da massa inicial da amostra.

As análises térmicas com emprego das técnicas de termogravimetria (TG) e calorimetria exploratória diferencial (DSC), em atmosfera inerte de $\mathrm{N}_{2}$, para amostras da Tinta modificada com AMS-Base úmida-2 e curadas com um catalisador ácido, evidenciam um mínimo de três etapas em seu processo de decomposição térmica. Na faixa de temperaturas entre 25 a $225^{\circ} \mathrm{C}$, a perda de massa da tinta curada é praticamente nula, indicando a sua maior resistência à decomposição térmica em relação à Tinta base seca curada com Aradur. A perda de massa na faixa de temperaturas entre 225 a $388^{\circ} \mathrm{C}$ é de cerca de $33 \%$, significativamente menor do que a perda de massa verificada nas segunda e terceira etapas, somadas, da decomposição térmica da Tinta base seca curada com Aradur.

A curva DSC para a tinta modificada com AMS evidencia dois picos de fluxo de calor positivo em $267^{\circ} \mathrm{C}$ e $371^{\circ} \mathrm{C}$, além de dois ombros de fluxo de calor positivo nas temperaturas de $338{ }^{\circ} \mathrm{C}$ e $385^{\circ} \mathrm{C}$, demonstrando a ocorrência de processos exotérmicos nessa faixa de temperatura. O pico localizado em $267^{\circ} \mathrm{C}$ pode ser associado à decomposição térmica com reorganização estrutural nas cadeias poliméricas interpenetrantes. A perda de massa associada a este processo na tinta modificada com o AMS é menor do que $5 \%(\mathrm{~m} / \mathrm{m})$ 
quando comparada ao processo semelhante na Tinta base seca curada com Aradur 850. Esta ocorrência corrobora os resultados de FTIR que indicam um maior grau de formação de ligações cruzadas, tanto na cadeia do poliéter siloxano quanto na cadeia polimérica da resina epóxi novolaca e, eventualmente, entre as cadeias dos dois polímeros utilizados na produção das tintas modificadas com AMS curadas. O processo exotérmico correspondente ao pico em $371^{\circ} \mathrm{C}$ da curva DSC se associa à decomposição térmica do poliéter siloxano, pois a curva DSC obtida para o poliéter siloxano curado com o mesmo catalisador ácido apresenta um pico exotérmico na mesma temperatura.

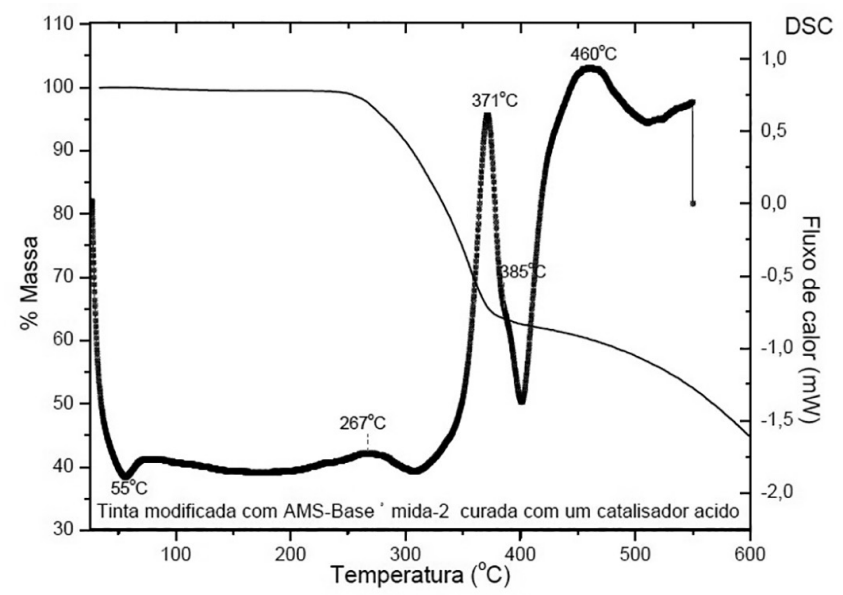

Figura 8. Curvas DSC e TG para a tinta modificada com AMS-Base úmida-2 curada com um catalisador ácido, em atmosfera de $\mathrm{N}_{2}$

A perda de massa observada para temperaturas maiores, entre 400 a $510^{\circ} \mathrm{C}$, com um pico exotérmico observado na temperatura de $460{ }^{\circ} \mathrm{C}$ na curva DSC da tinta, é de somente $4 \%$. Salienta-se, conforme citado anteriormente, que o processo que ocorre nessa faixa de temperatura pode ser associado à desidratação, com eliminação de água de grupos $-\mathrm{CH}_{2}-\mathrm{CH}(\mathrm{OH})-$ da resina epóxi novolaca e formação subsequente de ligações duplas. ${ }^{38} \mathrm{Com}$ isso, observa-se que a perda de massa na Tinta modificada com AMS é muito menor do que a perda de massa atribuída à decomposição térmica da Tinta base seca curada com Aradur 850, o que novamente corrobora as interpretações dadas aos resultados de FTIR.

Analogamente ao que se observou em temperaturas mais elevadas para a Tinta curada com o Aradur, a curva DTG da Tinta modificada com AMS também apresenta um pico em $595{ }^{\circ} \mathrm{C}$ associado à decomposição térmica de cadeias poliméricas aprisionadas na estrutura inorgânica formada. A massa residual na temperatura de $750{ }^{\circ} \mathrm{C}$ equivale a cerca de $35 \%$ da massa inicial da amostra.

\section{Medidas de Polarização Potenciodinâmicas e Espectroscopia de Impedância Eletroquímica (EIS)}

As curvas de polarização potenciodinâmicas obtidas para as superfícies do aço 1020 apenas jateada (Branco), e para as superfícies recobertas com a "Tinta base úmida" curada com catalisador ácido e com a "Tinta modificada com AMS-Base úmida-1" denominadas, respectivamente, de superfícies AM9 e AM11, estão apresentadas na Figura 10. Essas curvas foram obtidas a partir do potencial de circuito aberto (OCP), numa faixa de potencial $400 \mathrm{mV}$ menor e maior do que o OCP, com uma velocidade de varredura de potencial de $1 \mathrm{mV} \mathrm{s}^{-1}$.

As superfícies recobertas com as tintas desenvolvidas neste estudo apresentaram potenciais de corrosão (Ecorr) muito mais nobres (mais positivos) e densidades de corrente de corrosão (Jcorr) muito menores do que a superfície do aço carbono sem qualquer tipo de proteção. A

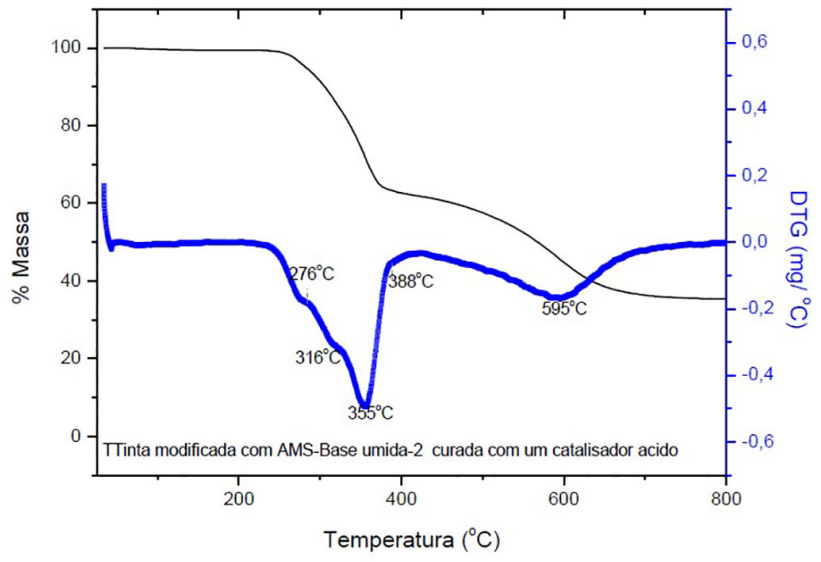

Figura 9. Curvas TG e DTG para a tinta modificada com AMS-Base úmida-2 curada com um catalisador ácido, em atmosfera de $\mathrm{N}_{2}$

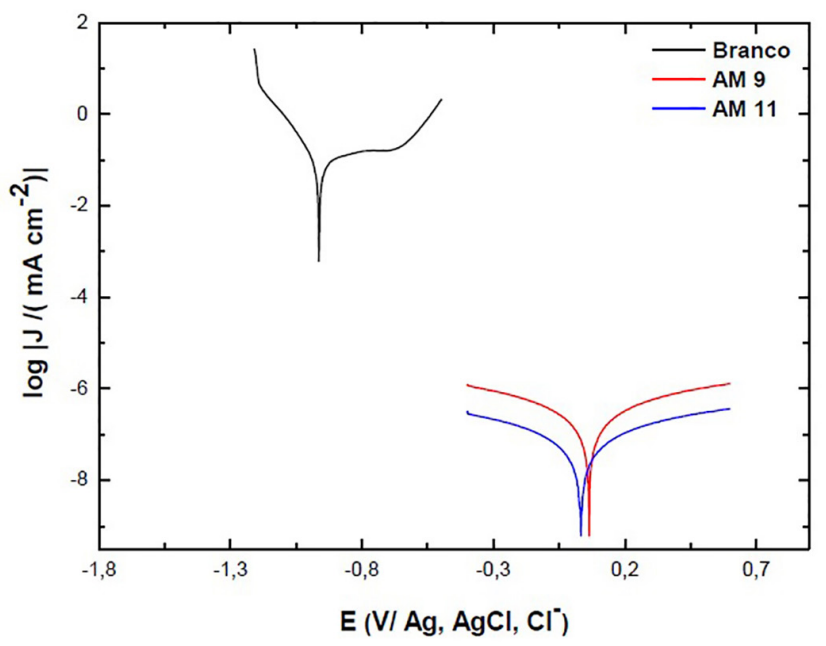

Figura 10. Curvas de polarização potenciodinâmica obtidas para as superfícies de aço carbono 1020 apenas jateada (Branco) e para as superfícies recobertas com a "Tinta base úmida" curada com catalisador ácido (AM9) e com a "Tinta modificada com AMS-Base úmida-1" (AM11) em solução de $\mathrm{NaCl}$ 0,5 $\mathrm{mol} \mathrm{L}^{-1}$

Tabela 1 apresenta os parâmetros eletroquímicos obtidos a partir das curvas de polarização potenciodinâmica apresentadas na Figura 10.

Os resultados de impedância eletroquímica foram obtidos sobrepondo ao potencial de circuito aberto uma perturbação senoidal, com uma amplitude de $5 \mathrm{mV}$, numa faixa de frequência compreendida entre $10^{5}$ e $10^{-2} \mathrm{~Hz}$. Nesta condição de perturbação, os resultados obtidos apresentaram uma flutuação significativa, o que costuma ser observado para superfícies recobertas com filmes que apresentam uma impedância muito elevada. Nesses casos, a corrente elétrica que passa através da interface superfície recoberta/solução eletrolítica é muito baixa, o que pode provocar flutuações nos resultados medidos. ${ }^{22}$ Os diagramas de Bode (gráfico de log $|\mathrm{Z} / \mathrm{Ohm}|$ versus $\log (\mathrm{f} / \mathrm{Hz})$ ) obtidos para as superfícies do aço 1020 recobertas com

Tabela 1. Parâmetros eletroquímicos obtidos das curvas de polarização apresentadas na Figura 10

\begin{tabular}{ccc}
\hline Amostras & Ecorr $(\mathbf{V} / \mathbf{A g} / \mathbf{A g C l , C l})$ & Jcorr $\left(\mathbf{m A ~ c m} \mathbf{~ c m}^{-2}\right)$ \\
\hline Branco & $-0,88 \pm 0,11$ & $(7,3 \pm 2,6) 10^{-2}$ \\
AM 9 & $0,10 \pm 0,04$ & $(6,7 \pm 1,1) 10^{-8}$ \\
AM 11 & $0,04 \pm 0,01$ & $(1,6 \pm 0,4) 10^{-8}$ \\
\hline
\end{tabular}


duas das tintas desenvolvidas (Figura 11) são caracterizados por uma reta com inclinação próxima de -1 , na região de frequência que varia entre $100-0,01 \mathrm{kHz}$, e por uma reta horizontal na faixa de $0,01 \mathrm{kHz}$ a $0,01 \mathrm{~Hz}$, em que o valor constante de impedância pode ser tomado como uma medida da resistência dos filmes que recobrem as superfícies..$^{22,23}$

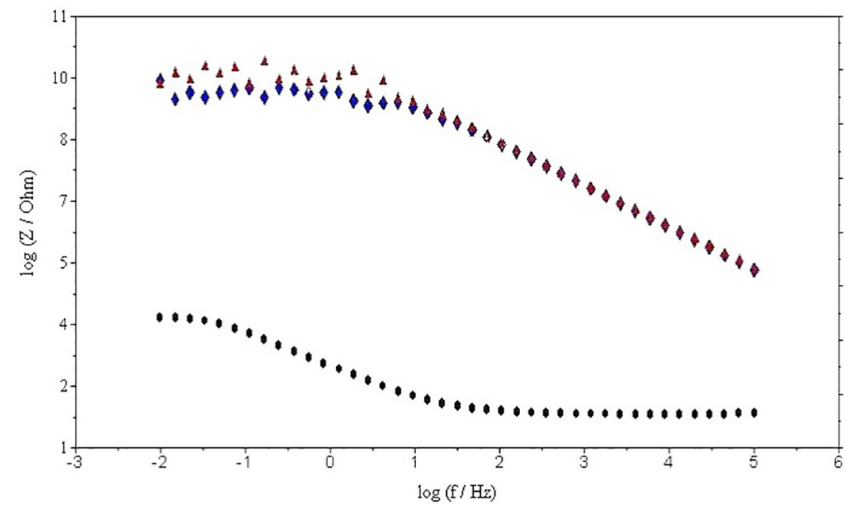

Figura 11. Gráfico de Bode para as amostras: Branco (๑), AM $9(\bullet)$ e AM 11 (A) expostas à solução de $\mathrm{NaCl} \mathrm{0,5} \mathrm{mol} \mathrm{L}^{-1}$

$\mathrm{O}$ valor de impedância, correspondente às retas observadas em regiões de baixas frequências nos diagramas de Bode, foi igual a $10^{4}$ $\mathrm{Ohm} \mathrm{cm}^{2}$ para a superfície sem recobrimento, e de $\sim 10^{10} \mathrm{Ohm} \mathrm{cm}^{2}$ para as superfícies AM9 e AM11. Os filmes das tintas desenvolvidas neste estudo apresentaram resistência uma ordem de grandeza maior quando comparadas a filmes de resinas epóxi modificadas com siloxanos em trabalhos disponíveis na literatura. ${ }^{39}$

As superfícies AM9 e AM11 foram submetidas a ensaios de imersão, de acordo com as Normas ASTM D1308-02 ${ }^{40}$ e ASTM D6943-03, ${ }^{41}$ em solução alcalina contendo $0,1 \mathrm{~mol} \mathrm{~L}^{-1}$ de $\mathrm{NaOH}$, em solução ácida contendo $0,1 \mathrm{~mol} \mathrm{~L}^{-1} \mathrm{de}_{2} \mathrm{SO}_{4}$ e em solução salina contendo $5 \%(\mathrm{~m} / \mathrm{m})$ de $\mathrm{NaCl}$, todas na temperatura ambiente, em água destilada, sem agitação, por $1600 \mathrm{~h}$. As superfícies não apresentaram alterações nos ensaios de imersão em meio ácido, alcalino e salino, indicando a resistência das tintas a meios ácidos e a meios alcalinos. Nos ensaios de imersão em água destilada a $30{ }^{\circ} \mathrm{C}$, as superfícies denominadas AM9 apresentaram um leve amarelamento após 672 h, indicando que, nesta temperatura e com exposição constante à água, a "Tinta base úmida" perde um pouco as suas propriedades de cor. Também, micrografias ópticas das superfícies não revelaram sinais de processo de corrosão após o período de imersão. Esta observação confirma a eficiência de proteção contra corrosão para o aço 1020, detectada nos resultados dos ensaios de corrosão apresentados.

Por outro lado, ampliando-se a escala de produção das tintas desenvolvidas para um processo de planta piloto, o uso do catalisador ácido na pré-hidrólise da mistura da resina epóxi com o poliéter siloxano acarretou resultados menos satisfatórios em relação à mistura e à qualidade do filme formado, apresentando pontos não enrijecidos que caracterizam ausência de reação (polimerização). Esse fato pode ser observado pela presença de ondulação em regiões de um determinado corpo de prova metálico onde o filme aplicado não curou. Com isso, na ampliação da escala de processo para produção em planta piloto, opta-se por utilizar uma poliamina aromática (Aradur 850) como agente de cura, devido ao fato de que as aminas são extremamente reativas, especialmente com resinas epóxi, produzindo filmes que tendem a proporcionar uma maior resistência químicas a diversas espécies consideradas quimicamente agressivas. Essa solução traz como vantagem a caracterização do produto obtido como uma tinta epóxi bi-componente de uso comercial.

\section{CONCLUSÕES}

Os espectros FTIR da mistura da resina epóxi novolaca EPN1179 com o poliéter siloxano, submetida à pré-hidrólise catalisada por ácido, e das tintas base seca e base úmida, curadas e não curadas, permitem concluir que ocorre a abertura parcial de anéis epóxi e o crescimento da cadeia polimérica da resina epóxi novolaca através de reação de homopolimerização, na qual um grupo C-OH do glicol, produzido na reação de adição de uma molécula de água a um anel epóxi protonado, liga-se a outro anel epóxi protonado, formando um éter de cadeia maior. Também há indícios de ocorrência da reação de condensação entre grupos silanóis $(\mathrm{Si}-\mathrm{OH})$ gerados na pré-hidrólise do poliéter siloxano e/ou entre estes e grupos $-\mathrm{CH}_{2}-\mathrm{OH}$ dos glicóis produzido na reação de homolimerização da resina epóxi. Os indícios de formação de ligações cruzadas entre os grupos -Si-OH e os grupos nas estruturas poliméricas formadas são ainda mais fortes nos espectros FTIR das tintas curadas. No caso das tintas modificadas com AMS-Base seca e Base úmida-2, não curadas e curadas, contendo $\mathrm{SiO}_{2}$ como carga adicional, o número de ligações cruzadas formadas dentro da cadeia do polisiloxano e o de ligações éter e de hidroxilas alifáticas na cadeia da resina epóxi é maior do que nas tintas base seca e base úmida curadas.

O comportamento térmico da "Tinta base seca" curada com a poliamina aromática evidencia a presença em excesso do poliéter siloxano na tinta preparada. Esse excesso é benéfico por sua capacidade de absorver quantidades residuais de umidade mantendo a integridade estrutural do revestimento. A "Tinta modificada com AMS-Base úmida-2" e curada com um catalisador ácido apresenta maior resistência à decomposição térmica do que a "Tinta base seca" curada com Aradur, fato que reforça os resultados de FTIR que apontam para um maior teor de ligações cruzadas nas cadeias do poliéter siloxano e da resina epóxi novolaca e, eventualmente, entre as cadeias dos dois polímeros utilizados na produção das Tintas modificadas com AMS curadas.

Superfícies do aço 1020 recobertas com as tintas desenvolvidas neste estudo apresentaram potenciais de corrosão (Ecorr) muito mais nobres (mais positivos) e densidades de corrente de corrosão (Jcorr) muito menores $\left(10^{-8} \mathrm{~mA} \mathrm{~cm}^{-2}\right)$ do que a superfície do aço carbono sem qualquer proteção tipo de proteção $\left(10^{-2} \mathrm{~mA} \mathrm{~cm} \mathrm{~cm}^{-2}\right)$. O valor significativamente menor de densidades de corrente de corrosão das superfícies recobertas com as tintas se deve à resistência elevada apresentada pelo filme de tinta recobrindo as superfícies (cerca de

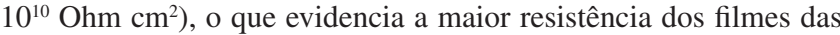
tintas desenvolvidas neste trabalho em comparação aos filmes de resinas epóxi modificadas com siloxanos nos trabalhos disponíveis na literatura.

Além disso, com a inclusão de uma poliamina aromática como agente de cura no processo em escala piloto de produção da tinta desenvolvida, o processo de cura foi aprimorado com a vantagem de o produto obtido se caracterizar, desta forma, como uma tinta epóxi bi-componente de uso comercial.

\section{AGRADECIMENTOS}

À Agência Nacional de Energia Elétrica (ANEEL) e à Électricité de France (EDF) pelo financiamento do projeto.

\section{REFERÊNCIAS}

1. Gu, H.; Ma, C.; Gu, J.; Guo, J.; Yan, X.; Huang, J.; Zhang, Q.; Guo, Z.; J. Mater. Chem. C 2016, 4, 5890.

2. Velan, T. V. T.; Bilal, I. M.; Def. Sci. J. 2002, 5, 87.

3. Liu, P.; Song, J.; He, L.; Liang, X.; Ding, H.; Li, Q.; Eur. Polym. J. 2008, 44,940 . 
4. Velan, T.V.T.; Bilal, I.M.; Bull. Mater. Sci. 2000, 3, 425.

5. Alagar, M.; Kumar, A. A.; Mahesh, K. P. O.; Dinakaran, K.; Eur. Polym. J. 2000, 36, 2449.

6. Ahmada, S.; Gupta, A. P.; Sharmin, E.; Alama, M.; Pandey, S. K.; Prog. Org. Coat. 2005, 54, 248.

7. Dean, K.; Cook, W. D.; Zipper, M. D.; Burchill, P.; Polymer 2001, 42, 1345.

8. Park, S. J.; Seo, M. K.; Lee, J. R.; J. Polym. Sci., Part A: Polym. Chem. 2000, 38, 2945.

9. Guo, B.; Jia, D.; Fu, W.; Qiu, Q.; Polym. Degrad. Stab. 2003, 79, 521. dd

10. Jin, F.-L.; Li, X.; Park, S.-J.; J. Ind. Eng. Chem. 2015, 29, 1.

11. Jeon, H. R.; Park, J. H.; Shon, M. Y.; J. Ind. Eng. Chem. 2013, 19, 849.

12. Das, G.; Kalita, R. D.; Deka, H.; Buragohain, A. K.; Karak, N.; Prog. Org. Coat. 2013, 76, 1103.

13. Kumar, K. S. S.; Biju, R.; Nair, C. P. R.; React. Funct. Polym. 2013, 73, 421.

14. Ji, W.-G.; Hu, J.-M.; Zhang, J.-Q.; Cao, C.-N.; Corros. Sci. 2006, 48, 3731 .

15. Ashok, K. A.; Alagar, M.; Rao, R. M. V. G. K.; Polymer 2002, 43, 693.

16. Devroey, D. R. E.; Homma, M.; Int. J. Adhes. Adhes. 2001, 21, 275.

17. Kumar, A. S.; Denchev, Z.; Alagar, M.; Eur. Polym. J. 2006, 42, 2419.

18. Kumar, A. S.; Sankara, T. S. N. N.; Prog. Org. Coat. 2002, 45, 323.

19. Kumar, A. S.; Balakrishnan, T.; Alagar, M.; Denchev, Z.; Prog. Org. Coat. 2006, 55, 207.

20. Farhadyar, N.; Rahimi A.; Langroudi, A. E.; Iran. Polym. J. 2005, 14, 155.

21. Peña-Alonso, R.; Rubio, F.; Rubio, J.; Oteo J. L.; J. Mat. Sci. 2007, 42, 595.

22. Frutuoso, A. G.; Rocco, J. A. F. F.; Oliveira, M. A. S.; Oliveira Junior, R. P.; INPI-PI 1.000.358-4 A2, 2011.

23. Oliveira, M. A. S.; Oliveira Junior, R. P.; Frutuoso, A.G.; Rocco, Relatório de projeto TMU pesquisa básica ANEEL, 2010.
24. Sassi, N.; Bureau, J. C.; Bakkali, A.; Vib. Spectrosc. 2002, 28, 299

25. Murthy, S.K.; Gleason, K. K.; Macromolecules 2002, 35, 1967.

26. Ahmad, Z.; Al Sagheer, F.; Al Arbash, A.; Ali, A.A.M.; J. Non-Cryst. Solids 2009, 355, 507.

27. Nocun, M.; Sowulski, S.; Leja, E.; Jedlinski, J.; Opt. Mater. 2005, 27, 1523.

28. Manocha, S. M.; Vashistha, D. Y.; Manocha, L. M.; J. Mater. Sci. Lett. 1997, 16, 705 .

29. Fidalgo, A.; Ilharco, L. M.; J. Non-Cryst. Solids 2001, 283, 144.

30. Pan, G.; Du, Z.; Zhang, C.; Li, C.; Yang, X.; Li, H.; Polymer 2007, 48, 3686.

31. Cherian, A. B.; Alen L. V.; Thachil, E. T.; Eur. Polym. J. 2007, 43, 1460.

32. Poljanšek, I.; Krajnc, M.; Acta Chim. 2005, 52, 238.

33. Karayannidou, E. G.; Achilias, D. S.; Sideridou, I. D.; Eur. Polym. J. 2006, 42, 3311.

34. Ramírez, C.; Rico, M.; Torres, A.; Barral, L.; López, J.; Montero, B.; Eur. Polym. J. 2008, 44, 3035 .

35. Manocha, S. M.; Vashistha, D. Y.; Manocha, L. M.; J. Sol-Gel Sci. Technol. 1999, 16, 221.

36. Manocha, L. M.; Yasuda, E.; Tanabe, Y.; Manocha, S.; Vashistha, D.; Bull. Mater. Sci. 2000, 23, 1.

37. Thompson, W. R.; Ho, M. C. M.; Pemberton, J. E.; Langmuir 1997, 13, 2291.

38. Ivnkovic, M.; Brnardic, I.; Ivankovic, H.; Huskic, M.; Gajovic, A.; Polymer 2009, 50, 2544.

39. Akbarinezhad, E.; Rezaei, F.; Neshati, J.; Prog. Org. Coat. 2008, 61, 45.

40. ASTM D1308-02, Effect of household chemicals on clear and pigmented organic finishes, ASTM International Standart, West Conshohocken, PA, 2007.

41. ASTM D6943-03, Immersion testing of industrial protective coatings, ASTM International Standart, West Conshohocken, PA, 2003. 